\title{
Identificação de anfetamina em amostras de cabelo por imunofluorescência polarizada
}

\author{
Saulo Rios Mariz ${ }^{1 *}$, Ovandir Alves Silva ${ }^{2}$ \\ ${ }^{1}$ Departamento de Farmácia, Universidade Federal do Maranhão, ${ }^{2}$ Faculdade de Ciências Farmacêuticas, \\ Universidade de São Paulo
}

*Correspondência:

S. R. Mariz

R. dos Duques, Bloco 01, .Ap. 103

65040-140 - Parque dos Nobres, São

Luís - MA - Brasil

E-mail: sjmariz22@hotmail.com
O uso indevido de anfetaminas tem preocupado as autoridades sanitárias em todo o mundo. No Brasil, destacam-se os anorexígenos anfetamínicos como o femproporex, que, no organismo, se biotransforma em anfetamina. Apesar de ser controlado por legislação específica, este fármaco tem sido amplamente utilizado em nosso país. Nas análises toxicológicas para verificação do uso de fármacos e drogas de abuso, têm-se empregado diferentes amostras biológicas. Mais recentemente a utilização do cabelo tem sido preconizada principalmente por informar sobre um uso a longo prazo da substância. A técnica para identificação de anfetaminas em cabelo é a cromatografia em fase gasosa acoplada à espectrometria de massas (CG-EM). A partir de um método descrito na literatura foram desenvolvidos estudos para avaliação da imunofluorescência polarizada como técnica de triagem na identificaçao de anfetamina em cabelo de usuários de anfetamínicos. Os resultados obtidos indicam que o método otimizado pode ser utilizado como triagem na identificação de anfetamina em cabelo.
Unitermos:

- Anfetaminas

- Análise de cabelo

- Imunofluorescência polarizada

- Femproporex

\section{INTRODUÇÃO}

A anfetamina e seus derivados são fármacos estimulantes do sistema nervoso central causadores de farmacodependência. No Brasil, é grande o uso indevido de anorexígenos anfetamínicos (Bell et al. 2001; INCB,1998; Nappo,1992), destacando-se o femproporex ou cianoetilanfetamina (Moffat et al., 1986). Vários trabalhos demonstram que até $70 \%$ de uma dose de femproporex pode se biotransformar no organismo, em anfetamina livre (Cody, 2002; Kraemer, Maurer, 2002; Musshoff, 2000; Cody, Valtier, 1999; Cody, Valtier, 1996;
Sznelwar, 1975). Apesar disso e de ser controlado por legislação específica (Brasil, 1997), vários trabalhos demonstram que este fármaco tem sido amplamente utilizado, não somente como inibidor do apetite (Halpern, Mancini, 2003; Rodrigues et al., 2002), mas também como estimulante da vigília (INCB, 1998; Silva et al., 1998; Lopes et al., 1997; Nappo, Oliveira, Morosini, 1994; Nappo, 1992).

A aplicação de métodos analíticos para identificação de psicofármacos em amostras biológicas tem se constituído importante instrumento colaborador no controle do uso de tais substâncias. A amostra de eleição para verifi- 
cação do uso recente é a urina, sendo que algumas técnicas são citadas na literatura para identificação de femproporex e seu produto de biotransformação (anfetamina) neste espécime biológico (Kraemer et al. 2000, Marson et al., 2000, Cody, Valtier, 1999, Cody, Valtier, 1996; Sznelwar, 1975).

Nos últimos anos, o cabelo tem sido estudado e utilizado como amostra na pesquisa de psicoativos, com propósitos distintos, pelo fato de fornecer informações sobre o uso da substância a longo prazo (Spinelli, 1999; Huestis, Cone, 1998; Sachs, Kintz, 1998; Cairns, Kippenberger, Gordorn, 1997; Sachs, 1997; Society of Hair Testing, 1997;).

A anfetamina e o femproporex se incorporam ao cabelo, embora em menor intensidade que outros anfetamínicos (Nakahara, Kikura, 1996; Luri, Nakahara, 1993). A identificação de anfetaminas em cabelo pode ser efetuada mediante várias técnicas de acordo com a finalidade da análise. Entre as técnicas de triagem, destacam-se os imunoensaios como a imunofluorescência polarizada (IFP). Entre as técnicas de confirmação, os resultados obtidos com a cromatografia em fase gasosa acoplada à espectrometria de massas (CG/EM) são inquestionáveis (Nakahara, 1995).

O emprego da imunofluorescência polarizada como técnica de triagem na identificação do uso de psicoativos em amostras biológicas tem crescido em função de características como: baixo custo, simplicidade e rapidez de execução. Apesar de alguns poucos trabalhos terem sido apresentados para identificação de anfetamina em cabelo (Kintz, Ludes, Mangin, 1992), até o momento, nenhum foi encontrado relatando a identificação de femproporex e/ou anfetamina em cabelo de usuários de femproporex.

O presente estudo mostra os resultados obtidos na otimização de um método para identificação de anfetamina em cabelo por IFP, a partir do originalmente proposto por Kintz, Ludes, Mangin (1992), bem como sua aplicação em amostras de cabelo de usuários de anfetaminas e de femproporex.

\section{MATERIAL E MÉTODOS}

\section{Soluções-padrão}

Soluções de femproporex e de sulfato de anfetamina, a $0,01 \%$ em metanol P.A. (Merck).

Soluções-padrão de femproporex a $500 \mathrm{ng} / \mathrm{mL}$ e 1000 ng/mL em tampão pH 7,5 (TDx FLx - ABBOTT)

Solução-padrão de anfetamina, preparada em tampão pH 7,5, a partir de controles $(500,1500$ e $4000 \mathrm{ng} /$ $\mathrm{mL})$ e calibradores $(1000,3000$ e $8000 \mathrm{ng} / \mathrm{mL})$ do kit reagente (TDx FLx - ABBOTT).

\section{Amostras}

Duas amostras de cabelo provenientes de usuários de femproporex, sexo feminino, com idade de 30 e 28 anos, respectivamente.

Duas amostras de cabelo provenientes de usuários de anfetaminas.

Pool de amostras de cabelo de não, usuários de anfetamínicos.

\section{Otimização da IFP para pesquisa de anfetamina em cabelo}

Para avaliar a possibilidade de o femproporex interferir na pesquisa de anfetamina por IFP (TDxFLxABBOTT) foram analisados $100 \mathrm{~mL}$ de soluções de femproporex em tampão nas concentrações de $500 \mathrm{ng} / \mathrm{mL}$ e $1000 \mathrm{ng} / \mathrm{mL}$. Como referência negativa foram utilizados $100 \mathrm{ml}$ do tampão.

Foram feitas modificações ao método originalmente proposto por Kintz, Ludes, Mangin (1992) para identificação de anfetamina em cabelo por imunofluorescência polarizada, tais como: a mudança na temperatura e tempo de digestão da amostra de cabelo para $40{ }^{\circ} \mathrm{C} / 18 \mathrm{~h}$ e o uso de uma solução-padrão de anfetamina, preparada a partir de controles e calibradores do equipamento (TDxFLxABBOTT).

Foi preparado um pool de cabelo cortando-se amostras de cabelo de não usuários de anfetamínicos, em pedaços de menor tamanho possível com posterior homogeneização.

Foi construída uma curva de calibração com adicionados de anfetamina em cabelo (pool) nas concentrações de 2,$96 ; 5,93 ; 8,90$ e $11,87 \mathrm{ng} / \mathrm{mg}$.

Alguns parâmetros de confiança do método foram avaliados a partir de resultados obtidos com adicionados de anfetamina em cabelo preparados de modo semelhante à curva de calibração. A exatidão foi avaliada de maneira aproximada, considerando-se a indisponibilidade de padrões secundários ou controles certificados de anfetamina em cabelo. Este parâmetro foi obtido pelo cálculo do percentual de inexatidão aproximada. A repetitividade foi obtida a partir do cálculo do coeficiente de variação (percentual) entre os resultados (em quintuplicata) de cada concentração em uma mesma corrida analítica. A reprodutibilidade foi avaliada calculandose o coeficiente de variação (percentual) entre as médias aritiméticas dos resultados (em triplicata) de cada concentração adicionada em corridas analíticas diferentes (Inmetro, 1995). 


\section{Aplicação da metodologia otimizada em amostras de cabelo de usuários de femproporex e de anfetaminas}

Após o registro de informações sobre o período de uso do fármaco, as amostras provenientes de usuários de femproporex (amostras "1" e " 2 ") foram coletadas o mais próximo possível do couro cabeludo, da parte posterior da cabeça. Os fios foram agrupados, sendo identificada a ponta correspondente à raiz. As amostras foram segmentadas considerando-se uma razão de crescimento capilar de $1,5 \mathrm{~cm}$ ao mês e cada segmento devidamente identificado em função do período de uso de femproporex relatado por indivíduo. Os segmentos foram cortados com tesoura em pedaços de menor tamanho possível das quais se tomou uma alíquota de $100 \mathrm{mg}$ para análise.

As amostras provenientes de usuários de anfetaminas (amostras " 3 " e " 4 ") foram cortadas com tesoura em pedaços de menor tamanho possível, de das quais se tomou, para análise, uma alíquota inferior a $100 \mathrm{mg}$, em função da disponibilidade.

Como referências negativas foram utilizadas alíquotas de $100 \mathrm{mg}$ do pool de cabelo. Como referências positivas foi adicionou-se anfetamina em alíquotas de 100 $\mathrm{mg}$ do pool de cabelo nas concentrações de 4 e $10 \mathrm{ng} / \mathrm{mg}$.

A identificação de anfetamina por imunofluorescência polarizada em cada alíquota foi feita conforme ilustrado no fluxograma (Figura 1). A concentração de $2 \mathrm{ng} / \mathrm{mg}$ foi utilizada como valor de corte.

\section{RESULTADOS E DISCUSSÃO}

\section{Otimização da IFP para pesquisa de anfetamina em cabelo}

O resultado negativo para a pesquisa de anfetamina por imunofluorescência polarizada em soluções de femproporex (500 ng/mL e $1000 \mathrm{ng} / \mathrm{mL}$ ) evidenciou que, nas condições estudadas, este fármaco não produziu reação cruzada na análise, sendo assim, a anfetamina, enquanto produto de biotransformação do femproporex, foi considerada como único analito a ser pesquisado para identificação deste fármaco por IFP.

Em relação ao método originalmente proposto por Kintz, Ludes, Mangin, (1992) a mudança na temperatura e tempo de digestão da amostra de cabelo para $40^{\circ} \mathrm{C} / 18 \mathrm{~h}$ apresentou resultados positivos na identificação de anfetamina por IFP em alíquotas de pool de cabelo adicionados com solução-padrão de anfetamina. Considerando que, em tentativas anteriores de reproduzir o método original, foram obtidos resultados negativos para pesquisa

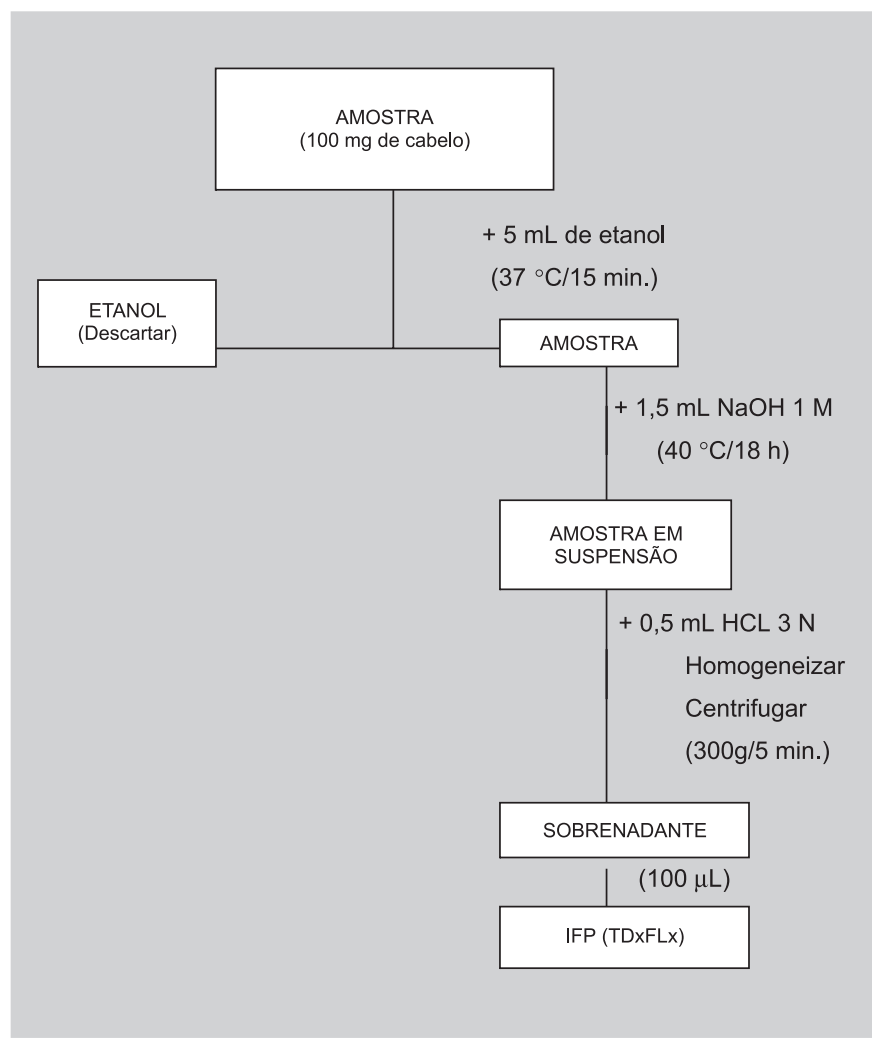

FIGURA 1 - Fluxograma do método de Kintz, Ludes, Mangin (1992) modificado, para pesquisa de anfetamina em cabelo por imunofluorescência polarizada.

de anfetamina em amostras adicionadas com este fármaco, tornou-se possível observar que a temperatura de $100{ }^{\circ} \mathrm{C}$ para digestão da amostra promovia perdas do analito suficientes para prejudicar sua identificação.

A solução-padrão de anfetamina, utilizada para preparo de adicionados em cabelo e preparada a partir de controles e calibradores do Kit reagente, apresentou concentração média de $2.374 \mathrm{ng} / \mathrm{mL}$ com cv\% $\%$ 6,28, valor dentro do limite estabelecido pelo fabricante do equipamento (Abbott Laboratories, 1996).

Os valores médios de cada ponto (em triplicata) da curva de calibração estão apresentados na Tabela I. A Figura 2 mostra que a intensidade da polarização é inversamente proporcional à concentração adicionada, produzindo uma curva típica da imunofluorescência polarizada.

Quanto aos parâmetros de confiança do método, inicialmente a Tabela II mostra que o método apresentou resultados de exatidão aproximada satisfatórios. Apenas a concentração de $2,96 \mathrm{ng} / \mathrm{mg}$ apresentou resultados de inexatidão aproximada superiores a $20 \%$, máximo preconizado para a técnica. Fato compreensível, pois a concentração adicionada neste ponto, se considerada em ng/mL, 
é de $118,4 \mathrm{ng} / \mathrm{mL}$, valor muito próximo ao limite de sensibilidade da técnica, que é de $100 \mathrm{ng} / \mathrm{mL}$ (Abbott Laboratories, 1996).

Tanto os estudos de repetitividade quanto os de reprodutibilidade (Tabelas III e IV, respectivamente) apresentaram resultados dentro do aceitável para a pesquisa de anfetamina por imunofluorescência polarizada (Abbott Laboratories, 1996).

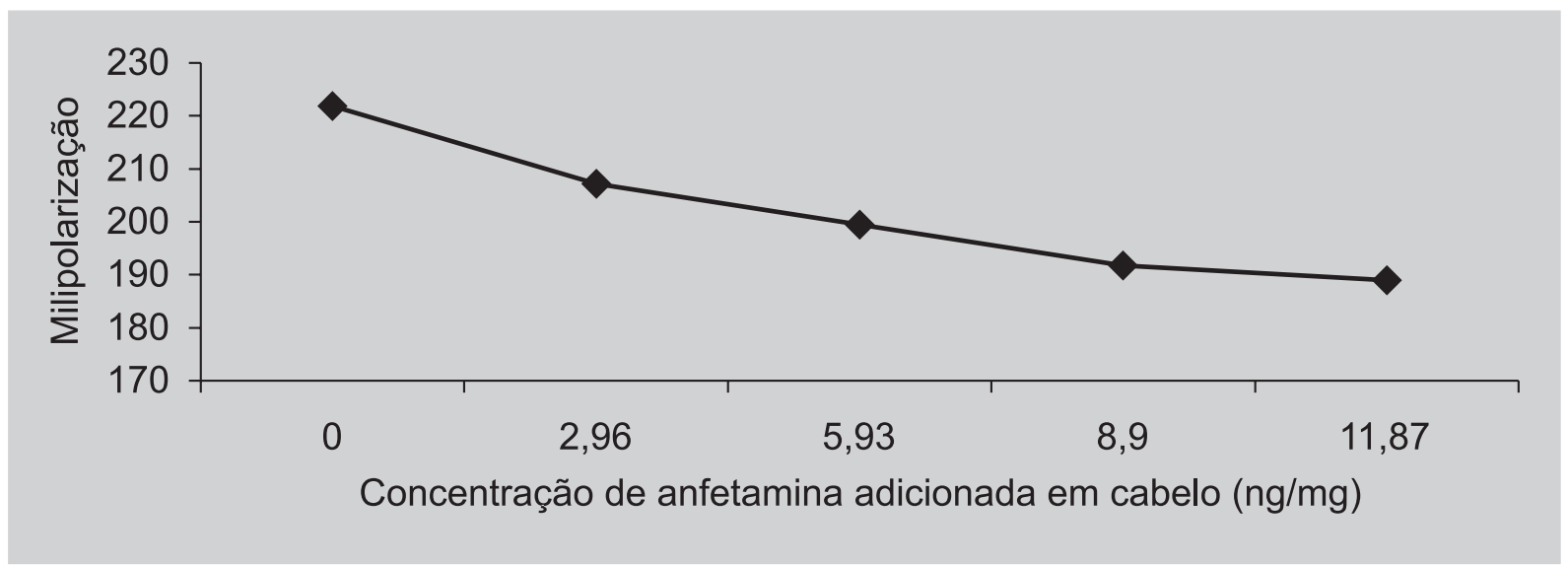

FIGURA 2 - Curva de calibração com adicionados de anfetamina em cabelo.

TABELA I - Valores (em milipolarização e ng/mg) dos adicionados de anfetamina em cabelo para preparo da curva de calibração

\begin{tabular}{ccc}
\hline $\begin{array}{c}\text { Concentração } \\
\text { Adicionada } \\
(\mathrm{ng} / \mathrm{mg})\end{array}$ & $\begin{array}{c}\text { Milipolarização } \\
(\mathrm{n}=3)\end{array}$ & $\mathrm{ng} / \mathrm{mg}$ \\
\hline 2,96 & 207,22 & 2,24 \\
5,93 & 199,51 & 5,24 \\
8,90 & 191,71 & 7,96 \\
11,87 & 188,94 & 11,25 \\
Referência Negativa & 221,81 & Negativo \\
\hline
\end{tabular}

TABELA II - Inexatidão aproximada da metodologia para identificação de anfetamina em cabelo

\begin{tabular}{ccc}
\hline $\begin{array}{c}\text { Concentração } \\
\text { Adicionada } \\
(\mathrm{ng} / \mathrm{mg})\end{array}$ & $\begin{array}{c}\mathrm{n} / \mathrm{mg} \\
(\mathrm{n}=3)\end{array}$ & $\begin{array}{c}\text { Resultado } \\
\text { Inexatidão aproximada } \\
\%\end{array}$ \\
\hline 2,96 & 2,28 & 23 \\
5,93 & 5,25 & 11,5 \\
8,90 & 8,13 & 8,7 \\
11,87 & 11,27 & 5,1 \\
\hline
\end{tabular}

TABELA III - Repetitividade (expressa em cv\%) da metodologia para identificação de anfetamina em cabelo

\begin{tabular}{|c|c|c|}
\hline $\begin{array}{l}\text { Concentração } \\
\text { Adicionada } \\
\text { (ng/mg) }\end{array}$ & $\begin{array}{l}\text { Resultado } \\
\text { (ng/mg) }\end{array}$ & $\begin{array}{c}\text { Coeficiente de } \\
\text { variação }(\%)\end{array}$ \\
\hline 2,96 & $\begin{array}{l}2,26 \\
2,28 \\
2,45 \\
2,11 \\
2,29\end{array}$ & 5,3 \\
\hline 5,93 & $\begin{array}{l}4,72 \\
5,16 \\
5,04 \\
5,08 \\
4,81\end{array}$ & 3,8 \\
\hline 8,90 & $\begin{array}{l}8,03 \\
8,02 \\
8,01 \\
8,16 \\
8,19\end{array}$ & 8,7 \\
\hline 11,87 & $\begin{array}{l}10,97 \\
11,03 \\
11,14 \\
11,03 \\
11,16\end{array}$ & 5,1 \\
\hline
\end{tabular}


TABELA IV - Reprodutibilidade (expressa em cv\%) da metodologia para identificação de anfetamina em cabelo

\begin{tabular}{ccc} 
(ng/mg) & & \\
\hline $\begin{array}{c}\text { Concentração } \\
\begin{array}{c}\text { Adicionada } \\
(\mathrm{ng} / \mathrm{mg})\end{array}\end{array}$ & $\begin{array}{c}\text { Média dos } \\
\text { resultados } \\
\text { (de cada ensaio) }\end{array}$ & $\begin{array}{c}\text { Coeficiente de } \\
\text { variação (\%) }\end{array}$ \\
\hline 2,96 & 2,28 & 0,65 \\
& 2,23 & \\
& 2,25 & \\
5,93 & 4,97 & 2,73 \\
& 5,24 & \\
& 5,05 & 3,78 \\
8,90 & 8,12 & \\
& 7,96 & \\
& 8,56 & 0,78 \\
11,87 & 11,05 & \\
& 11,25 & \\
& 11,00 & \\
\hline
\end{tabular}

\section{Aplicação da metodologia otimizada em amostras de cabelo de usuários de femproporex e de anfetaminas}

A pesquisa de anfetamina por imunofluorescência polarizada nas amostras de cabelo de usuários de femproporex apresentou resultados positivos em apenas um dos segmentos analisados de cada amostra, considerando-se um valor de corte de $2 \mathrm{ng} / \mathrm{mg}$ (Society of hair testing, 1997). A amostra "1" apresentou concentração de $2,3 \mathrm{ng} / \mathrm{mg}$ no segmento correspondente ao período de uso relatado e resultado negativo nos demais segmentos. $\mathrm{Na}$ amostra "2", do mesmo modo, o segmento correspondente ao período no qual o uso foi relatado apresentou concentração de anfetamina de $2,07 \mathrm{ng} / \mathrm{mg}$.

Esses resultados, apesar de muito próximos do valor de corte, são significativos considerando-se que:

- A anfetamina, entre os anfetamínicos, possui uma das menores razão de incorporação ao cabelo (Nakahara, Kikura, 1996; Luri, Nakahara, 1993).

- Este fármaco estava presente nas amostras como produto de biotransformação do femproporex, ou seja, cerca de 56 a $70 \%$ da dose do fármaco precursor ( Cody, Valtier, 1996; Sznelwar, 1975).

- O fármaco foi usado pelos pacientes em dose terapêutica e por curto período de tempo (um mês).

A pesquisa de anfetamina por imunofluorescência polarizada nas amostras de cabelo de usuários de anfetaminas apresentou resultados positivos, mesmo utilizando-se quantidades de amostra menores que $100 \mathrm{mg}$ e procedendo-se a análise sem segmentação em função da falta de informação sobre o padrão de uso. As concentrações de anfetamina obtidas foram: amostra " 3 " (83,84 mg) $=2,78 \mathrm{ng} / \mathrm{mg}$ e amostra " 4 " $(98,28 \mathrm{mg})=2,57 \mathrm{ng} / \mathrm{mg}$.

Em cada análise, as referências positivas e negativas apresentaram resultados de acordo com o esperado.

Diante dos resultados obtidos, cremos ser possível afirmar que o método padronizado é eficiente como procedimento de triagem para identificação de anfetamina por imunofluorescência polarizada em amostras de cabelo de usuários de femproporex e/ou anfetaminas.

\section{CONCLUSÕES}

Os estudos de otimização demonstraram que as modificações no método proposto por Kintz, Ludes, Mangin (1992), para identificação de anfetamina em cabelo por imunofluorescência polarizada, apresentaram resultados satisfatórios

O método otimizado, ao ser aplicado para pesquisa de anfetamina em amostras de cabelo de usuários de femproporex, apresentou resultados positivos nos segmentos correspondentes ao período no qual o uso foi relatado. Ao analisar-se amostras de cabelo de usuários de anfetaminas o resultado foi positivo mesmo utilizando-se uma quantidade de amostra inferior à padronizada (100 mg).

\section{ABSTRACT}

\section{Amphetamine detection in hair samples by FPIA}

The amphetamine abuse is a preoccupation of public health authorities all over the world. In Brazil, anoretic drugs like fenproporex have been much used. Fenproporex is metabolically dealkylated to amphetamine in the human body. In spite of its legal control, it has been abused in the country. Different samples have been used to identify the drug in toxicological analyses. Hair samples have been proposed recently to identify and study the long-term use of the drug. CG-MG is the technique used to identify amphetamines in hair samples. Following a method proposed in specific literature, some studies have been developed to evaluate the application of the fluorescence polarization imunoassay (FPIA) to identify amphetamine in hair samples of fenproporex users. The results show that the standard method may be used as screening in the identification of amphetamine by FPIA in hair samples of fenproporex users.

UNITERMS Amphetamines. Hair analysis. FPIA. Fenproporex 


\section{REFERÊNCIAS BIBLIOGRÁFICAS}

ABBOTT LABORATORIES. Diagnostics Division. $T D x^{\circledR /}$ TDxFL $x^{\circledR}$ assays manual: amphetamine/ methamphetamine II - List n ${ }^{\circ}$ 1A99. [s.L.p.] 1996. 8p. [66-6428/R1 Jan. 1996].

BELL, R. R.; CROOKHAM, S. B.; DUNN, W. A.; GRATES, K. M.; REIBER, T. M. A contemporaneous finding of fenproporex in a polydrug suicide. J. Anal. Toxicol. Niles, v.25, n.7, p.652-656, 2001

BRASIL. Leis, Decretos, etc. Portaria $\mathrm{n}^{\circ} 103$, de 10 mar. 1997. Diário Oficial da União, Brasília, n.47, 11 mar. 1997. Seção 1.

CAIRNS, T.; KIPPENBERGER, D. J.; GORDORN, A. Hair analysis for detection of drugs of abuse. In: WONG, S. H. Y.; SUNSHINE, I., eds. Analytical therapeutic drug monitoring and toxicology. Boca Raton: CRC Press, 1997. p.237-251.

CODY, J. T. Precursor medications as a source of methamphetamine and/or amphetamine positive drug testing results. J. Occup. Environ. Med., Baltimore, v.44, n.5, p.435-450, 2002.

CODY, J. T.; VALTIER, S. Detection of amphetamine following administration of fenproporex. J. Anal. Toxicol., Niles, v. 20, p 425-431,1996.

CODY, J. T.; VALTIER, S. Amphetamine and fenproporex levels following multidose administration of fenproporex. J. Anal. Toxicol., Niles, v. 23, p 187-194,1999.

HALPERN, A.; MANCINI, M. C. Treatment of obesity: na update on anti-obesity medications. Obes. Rev., Oxford, v. 4, n.1, p.25-42, 2003.

HUESTIS, M. A.; CONE, E. Alternative testing matrices. In: KARCH, S.B., ed. Drug abuse handbook. Boca Raton: CRC Press, 1998. p.817-821.

INMETRO - INSTITUTO NACIONALDEMETROLOGIA. Normalização e qualidade industrial. Vocabulário internacional de termos fundamentais e gerais de metrologia. Duque de Caxias, 1995. 52p.

INTERNATIONAL NARCOTIC CONTROL BOARD (Vienna). Psychotropic substances: statistics for 1996. New York: United Nations, 1998. 258p.
KINTZ, P.; LUDES, B.; MANGIN, P. Detection of drugs in human hair usin Abott ADx, with confirmation by gas chromatography/mass spectrometry (CG/MS). $J$. Forensic. Sci., Philadelphia, v.37, p.328-331, 1992.

KRAEMER, T.; MAURER, H. H. Toxicokinetics of amphetamines: metabolism and toxicokinetic data of designer drugs, amphetamine, methamphetamine, and their N-alkylderivatives. Ther. Drug Monit., Hagerstown, v.24, n.2, p.277-289, 2002.

KRAEMER, T.; THEIS, G. A.; WEBER, A. A.; MAURER, H. H. Studies on the metabolism and toxicological detection of the amphetamine-like anoretic fenproporex in human urine by gas chromatography-mass spectrometry and fluorescence polarization immunoassay. J. Chromatogr. B: Biomed. Appl., Amsterdam, v. 738, n.1, p.107-118, 2000.

LOPES, H. O.; PAIXÃO, H. H.; MONTEIRO, S. L.; PEDROSA, R. C. Formulações para emagrecimento: usos e abusos. Rev. Cienc. Farm., São Paulo, v.18, n.1, p.125-137, 1997.

LURI, K.; NAKAHARA, Y. Migração dos fármacos anfetamínicos (deprenyl, benzphetamine, femproporex, mefenorex ) do sangue para os pêlos. Yakubutsu Dô Tai, Tokyo, v.8, suppl., p.320, 1993.

MARSON, C.; SCHNEIDER, F.; MEYS, F.; WENNING, R. Structural elucidation of an uncommon phenylethylamine analogue in urine responsible for discordant amphetamine immunoassay results. J. Anal. Toxicol., Niles, v. 24, p.17-21, 2000.

MOFFAT, A. C.; JACKSON, J. V.; MOSS, M. S.; NIDDOP, B., eds. Clarck's isolation and identification of drugs. 2.ed. London: Pharmaceutical Press, 1986. p.349.

MUSSHOFF, F. Illegal or legitimate use ? Precursor compounds to amphetamine and methamphetamine . Drug Metab. Rev., New York, v. 32, n. 1, p. 15-44, 2000.

NAKAHARA, Y. Detection and diagnostic interpretation of amphetamines in hair. Forensic Sci. Int., Lausanne, v.70, p.135-153, 1995.

NAKAHARA, Y.; KIKURA, R. Hair analysis of drugs of abuse XIII. Effect of structural factors on incorporation of drugs into hair: the incorporation rates of amphetamine analogs. Arch. Toxicol., Berlin, v.70, p.841-849, 1996. 
NAPPO, S. A. Consumo de anorexígenos tipo anfetamina (dietilpropiona, mazindol e fenproporex) e de fenfluramina no Brasil. Prejuízo ou benefício para a saúde? J. Bras. Psiquiatr., Rio de Janeiro, v.41, n.8, p.417-421, 1992.

NAPPO, S. A.; OLIVEIRA, E. M.; MOROSINI, S. A prescrição por médicos brasileiros de fórmulas magistrais para emagrecer: uma duvidosa prática para saúde dos pacientes. Arq. Bras. Med., Rio de Janeiro, v.68, n.1, p.15-20, 1994.

\section{RODRIGUES, A. M.; RADOMINSKI, R. B.; SUPLICY, H.} L.; DE ALMEIDA, S. M.; NICLEWICZ, P. A.; BOGUSZEWSKI, C. L. The cerebrospinal fluid/serum leptin ratio during pharmacological therapy for obesity. $J$. Clin. Endocrinol. Metab., Bethesda, v.87, n.4, p.1621-6.

SACHS, H. History of hair analysis. Forensic Sci. Int., Lausanne, v.84, p.7-16, 1997.

SACHS, H.; KINTZ, P. Testing for drugs in hair: critical review of chromatographic procedures since 1992.J. Chromatogr. B: Biomed. Appl., Amsterdam, v.713, p.147-161, 1998.
SILVA, O. A.; YONAMINE, M.; ANTUNES, C. L. G.; GREVE, J. M. D.; MÍDIO, A. F. Fenproporex Abuse by Truck Drives in Brazil. In: SOFT-TIAFT 1998. Program and Abstracts. Albuquerque: Society of Forensic Toxicologists, International Association of Forensic Toxicologists,1998. Abstr. n.119, p.137.

SOCIETY of Hair Testing. Forensic Sci. Int., Lausanne, v.84, p.3-6, 1997.

SPINELLI, E. Mecanismos de incorporação de drogas no cabelo - revisão crítica da literatura. Rev. Bras. Toxicol. São Paulo, v. 12, n.1, p.1-8, 1999.

SZNELWAR, R. B. Chromatographic identification of amphetamine in the urine of patients treated with fenproporex. J. Eur. Toxicol., Paris, v.8, n.1, p.5-13, 1975.

Recebido para publicação em 07 de novembro de 2001. 